\title{
A NUTRIENT MIXTURE INHIBITS GLIOBLASTOMA XENOGRAFT U-87 MG GROWTH IN MALE NUDE MICE
}

\author{
M.W. Roomi, T. Kalinovsky, M. Rath, A. Niedzwiecki* \\ Dr. Rath Research Institute, 1260 Memorex Drive, Santa Clara, CA 95050, USA
}

\begin{abstract}
Background: Brain tumors are highly aggressive tumors characterized by secretions of high levels of matrix metalloproteinase-2 and $\mathbf{- 9}$, leading to tumor growth, invasion and metastasis by digesting the basement membrane and extracellular matrix components. We previously demonstrated the effectiveness of a nutrient mixture (NM) containing ascorbic acid, lysine, proline, and green tea extract in vitro: on activity of urokinase plasminogen activator, matrix metalloproteinases and TIMPs in various human glioblastoma (LN-18, T-98G and A-172) cell lines and on glioblastoma A-172 cell proliferation and Matrigel invasion. Aim: Our main objective in this study was to investigate the effect of the NM in vivo on human glioblastoma U-87 MG cell line. Materials and Methods: Athymic male nude mice inoculated with $3 \cdot 10^{6} \mathrm{U}-87 \mathrm{MG}$ cells subcutaneously and were fed a regular diet or a regular diet supplemented with $0.5 \%$ NM. Four weeks later, the mice were sacrificed, the tumors were weighed and measured. The samples were studied histologically. Results: NM inhibited tumor weight and tumor burden by $53 \%(p=0.015)$ and $48 \%(p=0.010)$, respectively. Conclusions: These results suggest the therapeutic potential of NM as an adjuvant in the treatment of glioblastoma. Key Words: glioblastoma U-87 MG cell line, nutrients, xenograft tumor growth.
\end{abstract}

Approximately 22,850 new cases and 15,320 deaths from brain and nervous system tumors were estimated in the United States for adults in 2015 [1]. Brain cancer (mainly gliomas and medulloblastomas) is the second (after leukemia) leading cause of cancer death in children aged under 20 , accounting for one third of all cancer deaths in this age group [2]. Though most brain tumors tend not to metastasize to distant areas outside the brain and/or spinal cord, they do tend to recur locally, or spread to other areas of the central nervous system. Tumor cell invasion depends upon degradation of the extracellular matrix, which, when intact, acts as a barrier to block cancer cell invasion [3, 4]. Rath and Pauling proposed a universal approach to tumor growth and expansion using nutrients such as lysine and ascorbic acid to target plasmin-mediated connective tissue degradation [5]. Subsequent studies confirmed this approach and led to identifying a novel formulation composed of lysine, ascorbic acid, proline and green tea extract and other micronutrients, which has shown significant anticancer activity against a large number ( $\sim 0)$ of cancer cell lines, blocking cancer growth, tissue invasion and matrix metalloproteinase (MMP) expression both in vitro and in vivo [6].

In a previous in vitro study, the nutrient mixture (NM) significantly inhibited urokinase plasminogen activator (UPA) and MMP secretion and increased TIMP-2 secretion in glioma cell lines [7]. Other studies found significant inhibition of cell proliferation and Matrigel invasion of glioma cell line A-172 by NM [8] and significant negative correlation between NM inhibition of Matrigel invasion and NM modulation of the MMP-2 activity of glioma cell line A-172 [9]. Our main objective in this study was to investigate the effect

Submitted: December 8, 2015.

*Correspondence: E-mail: author@drrath.com

Abbreviations used: MMP - matrix metalloproteinase; NM - nutrient mixture; uPA - urokinase plasminogen activator. of NM in vivo using human glioblastoma U-87 MG xenografts in male nude mice.

\section{MATERIALS AND METHODS}

Cell line and NM. Human glioblastoma cell line U-87 MG was obtained from ATCC (American Type Culture Collection, Rockville, MD, USA). The NM, prepared by VitaTech (Hayward, CA), was composed of the following ingredients in the amounts indicated: Vitamin $\mathrm{C}$ (as ascorbic acid and as $\mathrm{Mg}, \mathrm{Ca}$, and palmitate ascorbate) $700 \mathrm{mg}$; L-lysine $1000 \mathrm{mg}$; L-proline $750 \mathrm{mg}$; L-arginine 500 mg; N-acetyl cysteine 200 mg; standardized green tea extract ( $80 \%$ polyphenol) $1000 \mathrm{mg}$; selenium $30 \mu \mathrm{g}$; copper $2 \mathrm{mg}$; manganese $1 \mathrm{mg}$.

Animals. Male nude mice, approximately 5 weeks of age on arrival, were purchased from Simonsen Laboratories, Gilroy, CA, and maintained in microisolator cages under pathogen-free conditions on a 12hour light/12-hour dark schedule for a week. All procedures were performed according to humane and customary care and use of experimental animals and followed a protocol approved by internal institutional animal safety review committee.

Experimental design. After housing for a week, the mice $(n=12)$ were inoculated subcutaneously with $3 \cdot 10^{6}$ glioblastoma U-87 MG cells in $0.2 \mathrm{ml}$ PBS and $0.1 \mathrm{ml}$ Matrigel (BD Bioscience, Bedford, MA). After injection, the mice were randomly divided into two groups; the control group of mice was fed regular Laboratory Rodent Diet 5001 from Purina Mills, LLC (Gray Summit, MO, USA). and the NM group - the regular diet supplemented with $0.5 \% \mathrm{NM}(\mathrm{w} / \mathrm{w})$. The $0.5 \%$ NM diet was milled and pressed by Purina Mills, LLC and generated by Vitatech (Tustin, CA, USA). During the study, the mice consumed, on the average, $4 \mathrm{~g}$ of their respective diets per day. Thus, the supplemented mice received approximately $20 \mathrm{mg}$ of NM per day. After four weeks, the mice were sacrificed and their tumors were excised and processed for histology. Dimensions (length and width) of tumors were mea- 
sured using a digital caliper, and the tumor burden was calculated using the following formula: $0.5 \times$ length $\times$ width. Mean weight of mice at initiation of study and termination of study did not differ significantly between the groups.

Histology. Tissue samples were fixed in $10 \%$ buffered formalin. All tissues were embedded in paraffin and cut at 4-5 microns. Sections were deparaffinized through xylene and graduated alcohol series to water and stained with hematoxylin and eosin ( $H$ \& E) for evaluation using a standard light microscope.

Statistical analysis. The results were expressed as means \pm SD and analyzed by independent sample " $t$ " test using MedCalc software (Mariakerke, Belgium).

\section{RESULTS AND DISCUSSION}

NM strongly inhibited the growth of U-87 MG xenografts in male nude mice. Mean tumor weight was inhibited by $53 \%(p=0.0147)$ with NM $0.5 \%$ dietary supplementation, as shown in Fig. 1, $a$ and tumor burden was inhibited by $48 \%$ ( $p=0.0098$ ), as shown in Fig. $1, b$. There was no significant difference between mean body weights of groups of mice.
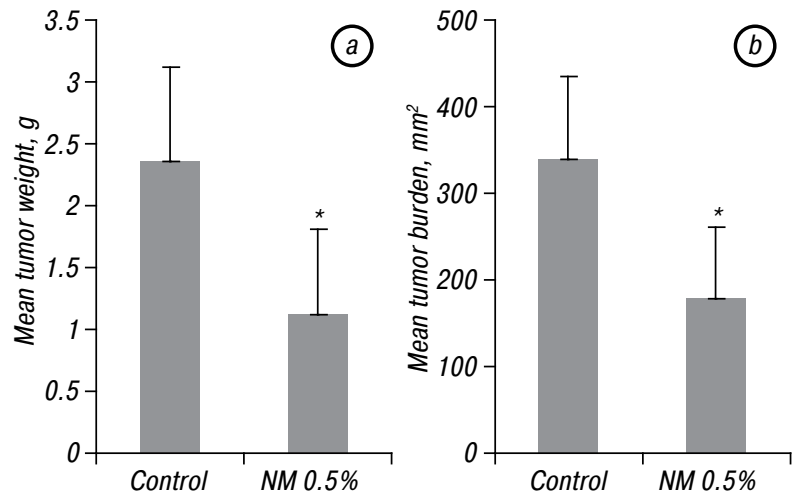

Fig. 1. Effect of NM on mean tumor weight (a) and tumor burden (b) in nude mice grafted with U-87 MG cells. * $p=0.015$ (a) and $\mathrm{p}=0.01(b)$ as compared to control

Histologically, the tumors from both groups were irregularly round, subcutaneous masses, composed of solid sheets and nests of irregularly round epithelioid cells with prominent round to ovoid nuclei and lightly staining bluish cytoplasm with ill-defined cell borders. Tumors from control and NM-supplemented mice were similar morphologically, but the tumors from supplemented mice were significantly smaller in size. Areas of tumor necrosis ranged from $50-80 \%$ of tumor mass in the control group compared to $10-60 \%$ in the NM group. Areas of tumor necrosis were associated with moderate to severe neutrophilic infiltration (Fig. 2, $a-d$ ).

Previous in vitro results from the cellular proliferation study of glioblastoma A-172 support the in vivo findings, as NM showed increased toxicity in cells in a dosedependent manner, with $50 \%$ inhibition of cell growth in cells exposed to $1000 \mu \mathrm{g} / \mathrm{ml} \mathrm{NM}$ and total block of invasion through Matrigel at $1000 \mu \mathrm{g} / \mathrm{ml} \mathrm{NM}$ [8]. In addition, prior studies of several glioblastoma cell lines, demonstrated potent, significant suppression of invasive parameters by the NM, including UPA and MMP-2 and -9 [7].

Brain cancer is the second leading cause of cancer death in children under 20 years, accounting for one third of all cancer deaths in this age group. Current treatment methods for gliomas are generally ineffective and toxic. Thus, there is a need for development of effective therapeutic agents for these cancers with minimal toxicity. Our studies demonstrated that the mixture of the non-toxic components of NM significantly inhibited the growth and tumor burden of glioblastoma cell line U-87 MG in vivo. These results in addition to our prior in vitro studies with glioblastoma cell lines, which documented significant inhibition of MMP-2 and-9 secretion and invasion by NM, suggest the potential of NM as an adjuvant in treatment of glioblastomas.
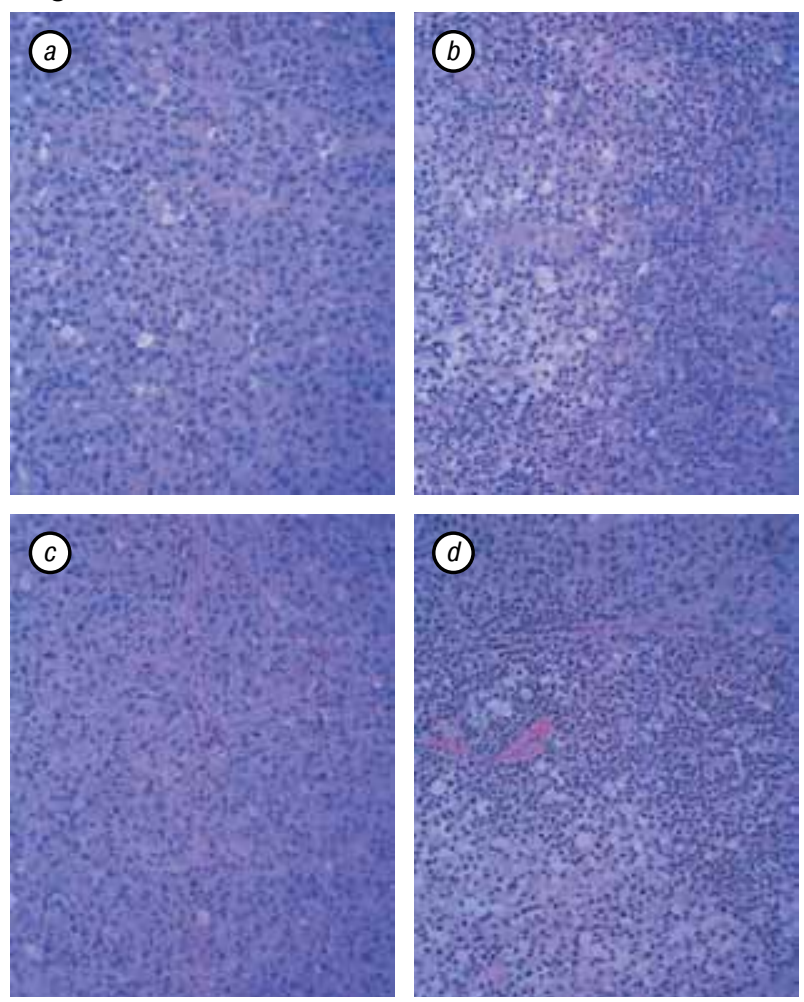

Fig. 2. Histopathology of tumors from control $(a, b)$ and NMsupplemented $(c, d)$ mice, $\times 200$. In $b$ and $d$ tumor necrosis and associated neutrophilic inflammation are present

\section{ACKNOWLEDGEMENTS}

The research study was funded by Dr. Rath Health Foundation (Santa Clara, CA, USA), a non-profit organization. We thank consulting pathologist Alexander de Paoli, DVM, PhD, IDEXX Reference Laboratories for providing histopathology slides.

\section{REFERENCES}

1. ACS. What are the key statistics about brain and spinal cord tumors? (http://www.cancer.org/cancer/braincnstumorsinadults/ detailedguide/brain-and-spinal-cord-tumors-in-adults-keystatistics).

2.ABTA(American Brain Tumor Association). Brain tumor facts (http://www.abta.org/about-us/news/brain-tumor-statistics/).

3. Yurchenko PD, Schitny JC. Molecular architecture of basement membranes. FASEB J 1990; 4: 1577-90. 
4. Barsky SH, Siegel GP, Jannotta F, et al. Loss of basement membrane components by invasive tumors but not by their benign counterparts. Lab Investig 1983; 49: 140-7.

5. Rath M, Pauling L. Plasmin-induced proteolysis and the role of apoprotein(a), lysine and synthetic analogs. Orthomol Med 1992; 7: 17-23.

6. Niedzwiecki A, Roomi MW, Kalinovsky T, et al. Micronutrient synergy - a new tool in effective control of metastasis and other key mechanisms of cancer. Cancer Metastasis Rev 2010; 29: 529-43.
7. Roomi MW, Kalinovsky T, Niedzwiecki A, et al. Modulation of UPA, MMPs and their inhibitors by a novel nutrient mixture in human glioblastoma cell lines. Int J Oncol 2014; 45: 887-94.

8. Roomi MW, Ivanov V, Kalinovsky T, et al. Inhibition of glioma cell line A-172 MMP activity and cell invasion in vitro by a nutrient mixture. Med Oncol 2007; 24: 231-8.

9. Roomi MW, Monterrey JC, Kalinovsky T, et al. Inhibition of invasion and MMPs by a nutrient mixture in human cancer cell lines: a correlation study. Exp Oncol 2010; 32: 243-8. 